\title{
Is There Any Correlation Between Preoperative Platelet Indices and Surgical Prognostic Factors in Patients with Cervical Cancer?
}

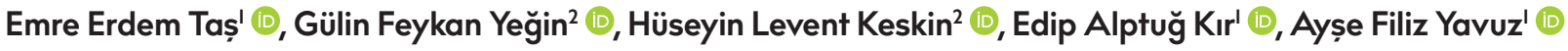 \\ 'Department of Obstetrics and Gynecology, Ankara Yıldırım Beyazıt University School of Medicine, Ankara, Turkey \\ ${ }^{2}$ Department of Obstetrics and Gynecology, Ankara Atatürk Training and Research Hospital, Ankara, Turkey \\ ORCID IDs of the authors: E.E.T. 0000-000I-6043-2700; G.F.Y. 0000-000I-8006-5055; H.L.K. 0000-0002-2268-382I; E.A.K. 0000-0002- \\ 2293-3624; A.F.Y. 0000-0003-3699-7757.
}

Cite this article as: Taș EE, Yeğin GF, Keskin HL, Kır EA, Yavuz AF. Is There Any Correlation Between Preoperative Platelet Indices and Surgical Prognostic Factors in Patients with Cervical Cancer?. Cyprus J Med Sci 2019; 4(I): 38-42.

\section{BACKGROUND/AIMS}

To evaluate the variations in platelet indices (PIs), including platelet count, mean platelet volume (MPV), platelet distribution width (PDW), and plateletcrit (PCT), in cervical cancer (CC) and to evaluate the relationship between these Pls and pathological features of tumor (i.e., tumor stage and size, depth of stromal invasion, lymphovascular space invasion (LVSI), and histological type).

\section{MATERIAL and METHODS}

This retrospective, comparative study included 40 patients with CC who underwent complete clinical staging and 80 healthy controls at a tertiary center between 2007 and 2017. Groups were compared using independent sample t-tests and Mann-Whitney U tests. Variables with a $p<0.05$ were included in the receiver operating characteristic curve analysis to determine cut-off values. The associations of Pls with pathological features were analyzed using the chi-square test.

\section{RESULTS}

The mean age of patients with CC was 56.9 years old. Demographic characteristics (i.e., age, gravidity, and parity) did not differ between the groups ( $p>0.05)$. Mean MPV and PCT were significantly lower $(p=0.002$ and $p=0.026$, respectively), and PDW was significantly higher $(p=0.049)$ in the CC group than in the control group. The platelet count did not differ significantly between the groups ( $p=0.558$ ). The optimal cut-off points of MPV, PDW, and PCT levels for discriminating between the groups were $10.45 \mathrm{fL}, 13 \mathrm{fL}$, and $0.28 \%$, respectively. Except a significant relationship between low PCT level and positive LVSI $(p=0.0 \mathrm{I})$, there was no significant relationship between Pls and pathological features.

\section{CONCLUSION}

Low MPV and PDW and high PCT may be considered as a useful additional tool for preoperative diagnosis of CC. However, the usability of these markers for predicting pathological features is unsatisfactory.

Keywords: Mean platelet volume, platelet count, prognosis, uterine cervical neoplasms

\section{INTRODUCTION}

Cervical cancer (CC) is the third most common malignancy of women worldwide, and approximately 266,000 patients die from this disease annually (I). However, the survival of patients with CC is well-correlated with the stage of tumor and other prognostic factors (i.e., tumor size, histological type and grade, depth of stromal invasion, lymphovascular space invasion (LVSI), and lymph node involvement status) (I).

Cervical cancer is staged clinically. The current staging procedure includes histological diagnosis by biopsy or cold-knife conization, pelvic examination under anesthesia, and related imaging techniques (i.e., cystoscopy, proctoscopy, and chest radiography). If needed, magnetic resonance imaging and computerized tomography can also be used. On the other hand, the uses of tumor markers (i.e., serum squamous cell carcinoma antigen, tissue polypeptide antigen, carcinoembriyonic antigen, and carcinoma antigen 125) for diagnosing, monitoring response to therapy, and detecting recurrence in CC have been investigated $(2,3)$. Nevertheless, none of them has achieved widespread acceptance, yet. 
It is well-known that inflammation influences the tumor microenvironment and plays a central role on the cancer development process (i.e., initiation, promotion, progression, and metastasis) (4). Simultaneously, tumor-induced inflammatory response may lead to change in complete blood count (CBC) parameters (5). In addition, some studies about CC revealed that various $C B C$ parameters have a significant relationship with CC and its pathological features (5-15). Furthermore, the utility of platelet indices (PIs) for patients with CC remains to be determined.

The aim of the present study was to evaluate the differences in Pls, including platelet count, mean platelet volume (MPV), platelet distribution width (PDW), and plateletcrit (PCT), in CC. Furthermore, we examined whether there was any relationship between these indices and various pathological features of tumor (i.e., tumor stage and size, depth of stromal invasion, LVSI status, and histological type).

\section{MATERIAL and METHODS}

This was a retrospective, case-control study conducted at a tertiary hospital. The research was conducted in accordance with the World Medical Association Declaration of Helsinki, revised in 2000, Edinburgh. The study was approved by the Ankara Atatürk Training and Research Hospital ethical review board committee of the institution (approval no.: 117). Written informed consent was obtained from all participants.

The medical records of 55 patients with CC who underwent complete clinical staging and the diagnosis of cancer as confirmed by histology in our institution between 2007 and 2017 were analyzed to form the CC group. The medical records of 80 healthy controls who underwent surgery for interval tubal sterilization or vaginal reconstruction at the same institution during the same time period were analyzed to form the control group. Exclusion criteria were as follows: concomitant malignancy, acquired or congenital hematological disease, acute or chronic inflammatory disease, use of any drug that may influence the coagulation cascade (e.g., anticoagulants, hormonal contraceptives, and steroids), and smoking. Fifteen patients from the CC group were excluded from the study. A total of 40 patients with CC and 80 healthy control patients were enrolled in the study.

Demographic (i.e., age, gravidity, and parity) and pathological (i.e., tumor stage and size, depth of stromal invasion, LVSI status, and histological type) features and preoperative Pls (i.e., platelet counts, MPV, PDW, and PCT levels) were obtained from the patients' medical records.

Blood samples were collected while patients were admitted to the hospital and before receiving any medications. Specimens were analyzed within $2 \mathrm{~h}$ using a Sysmex XE-2I00 Automated CBC Analyzer (Sysmex Europe, Germany).

The CC and control groups were compared with regard to the examined demographic characteristics and Pls. Once variables significantly associated with CC were determined, correlations between these variables with tumor stage (stage <IIB vs. $\geq I I B$ ), tumor size $(<4 \mathrm{~cm}$ vs. $\geq 4 \mathrm{~cm}$ ), depth of stromal invasion $(<50.0 \%$ vs. $\geq 50.0 \%$ ), and LVSI status (positive vs. negative) were investigated.

\section{Statistical Analyses}

Statistical analyses were conducted using Statistical Package for the Social Sciences for Windows, software ver. 2I.0 (IBM Corp., Armonk, NY, USA). The Kolmogorov-Smirnov test was used to assess the normality of data. Normally distributed data were expressed as mean and standard deviation. Nonparametric data were expressed as median and interquartile range (IQR). The independent samples t-test and Mann-Whitney $U$ test were used for comparison of the groups. Variables with a $p<0.05$ were included in the receiver operating characteristic (ROC) curve analysis to determine the cut-off values. Then, the largest Youden Index (sensitivity+specificity-I) was selected as the optimal cut-off point. The chi-square test or Fisher's exact test was used to analyze the associations of Pls with pathological features. Odds ratios and $95.0 \%$ confidence intervals were determined. A p value $<0.05$ was considered statistically significant.

\section{RESULTS}

The mean age of patients with CC was $56.9 \pm 15$ years. Medians of gravidity and parity were 4 (IQR: 3 ) and 3 (IQR: 3), respectively. Demographic characteristics (age, gravidity, and parity) did not differ significantly between the CC and control groups ( $p>0.05$ for all) (Table I).

Among the preoperative Pls, mean MPV and PCT were significantly lower ( $p=0.002$ and $p=0.026$, respectively), and PDW was significantly higher $(p=0.049)$ in the CC group than in the control group. The mean platelet count did not differ significantly between the groups $(p=0.55)$ (Table I). Furthermore, ROC curve analyses revealed that the optimal cut-off points of MPV, PDW, and PCT levels for discriminating between the CC and control groups were $10.45 \mathrm{fL}, 13 \mathrm{fL}$, and $0.29 \%$, respectively. ROC curve analyses of MPV, PDW, and PCT are shown in Figure I.

The pathological features of patients with $\mathrm{CC}$ and the relationship of MPV, PDW, and PCT with these are summarized in Table

\begin{tabular}{|c|c|c|c|}
\hline Variable & $\begin{array}{c}\text { Cervical cancer } \\
n=40(33.3 \%)\end{array}$ & $\begin{array}{c}\text { Control } \\
n=80(66.6 \%)\end{array}$ & $\mathrm{P}^{*}$ \\
\hline \multicolumn{4}{|c|}{ Demographic characteristic } \\
\hline Age (years), mean $\pm S D$ & $56.9 \pm 15$ & $51.9 \pm 9.8$ & 0.066 \\
\hline Gravidity, median (IQR) & $4(3)$ & $4(2.75)$ & 0.053 \\
\hline Parity, median (IQR) & $3(3)$ & $3(2)$ & 0.165 \\
\hline \multicolumn{4}{|l|}{ Platelet indices ${ }^{a}$} \\
\hline Platelet count $\left(\times 10^{3} / \mu \mathrm{L}\right)$ & $291.1 \pm 91.6$ & $300.4 \pm 77.5$ & 0.558 \\
\hline$M P \vee(f L)$ & $10.0 \pm 1.3$ & $10.6 \pm 0.8$ & 0.002 \\
\hline PDW (fL) & $13.5 \pm 2.6$ & $12.7 \pm 1.7$ & 0.053 \\
\hline Plateletcrit (\%) & $0.28 \pm 0.07$ & $0.31 \pm 0.07$ & 0.026 \\
\hline
\end{tabular}


2. Of the 40 patients, 26 (65.0\%) were at early stage (International Federation of Gynecology and Obstetrics stage $\leq \mathrm{IIB}$ ). However, tumor size was $\geq 4 \mathrm{~cm}$, and depth of stromal invasion was $\geq 50.0 \%$ in 18 ( $45.0 \%$ ) patients, and LVSI was positive in 13 (32.5\%) patients. The majority of histological type was squamous cell carcinoma (87.5\%). Except a significant relationship between lower PCT level and positive LVSI $(p=0.01)$, there was no significant relationship between Pls and CC's pathological features.

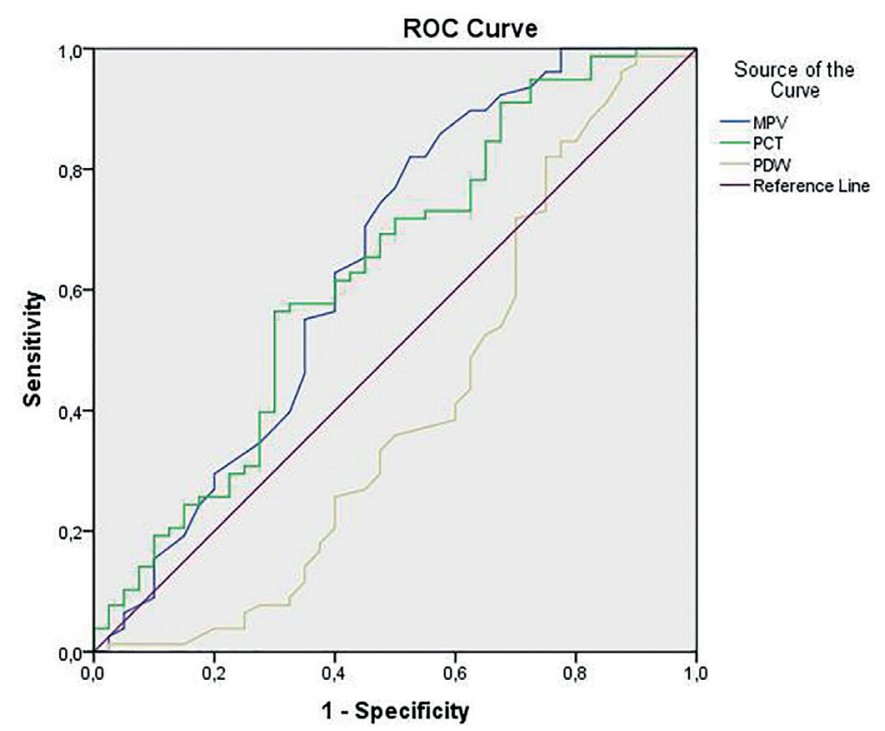

FIGURE I. Receiver operating characteristic curve analyses of mean platelet volume, platelet distribution width, and plateletcrit levels for discriminating patients with cervical cancer from control patients (area under curves were $0.64,0.59$, and 0.63 , respectively)

\section{DISCUSSION}

The present study has shown that some Pls, including MPV and PCT, were significantly lower, and that PDW was significantly higher in the CC group than in the control group. Nevertheless, it revealed that except a significant relationship between lower PCT level and positive LVSI, there were no significant relationships between Pls and CC's pathological features.

Cervical cancers occur and are diagnosed at an earlier age than other gynecological malignancies (I). In population-based studies from different regions worldwide, the median age of the patients has been reported to be between 44.7 and 52 years (16-18). In our study, the mean age of patients with CC was 56.9 years and a little more than these data. This may be because of the small sample size of our study population or may be a result of representing a single-center experience.

Platelets are dynamic cytoplasmic fragments of bone marrow megakaryocytes. In addition to their role in hemostasis and thrombosis, recently, increasing evidence displayed that platelets play a role on inflammatory processes, host defense, wound healing, angiogenesis, and remodeling (19). In addition to platelet count, Pls, including MPV, PDW, and PCT, are potential biomarkers of platelet activation. Various studies investigating the relationship between CCs and Pls have shown that these markers may correlate with the prognosis of disease (20-24).

High platelet count is a well-known systemic inflammatory response to the development of cancer (20). Various studies with CC showed that platelet count is significantly higher in patients with CC than in healthy controls (6). Furthermore, in a comprehensive review, Cheng et al. suggested that high platelet count may reflect poor prognosis (i.e., higher clinical stage, positive pelvic lymph node involvement, and a larger tumor size) (20). In

TABLE 2. The relationship of MPV, PDW, and plateletcrit with clinicopathological features of cervical cancer

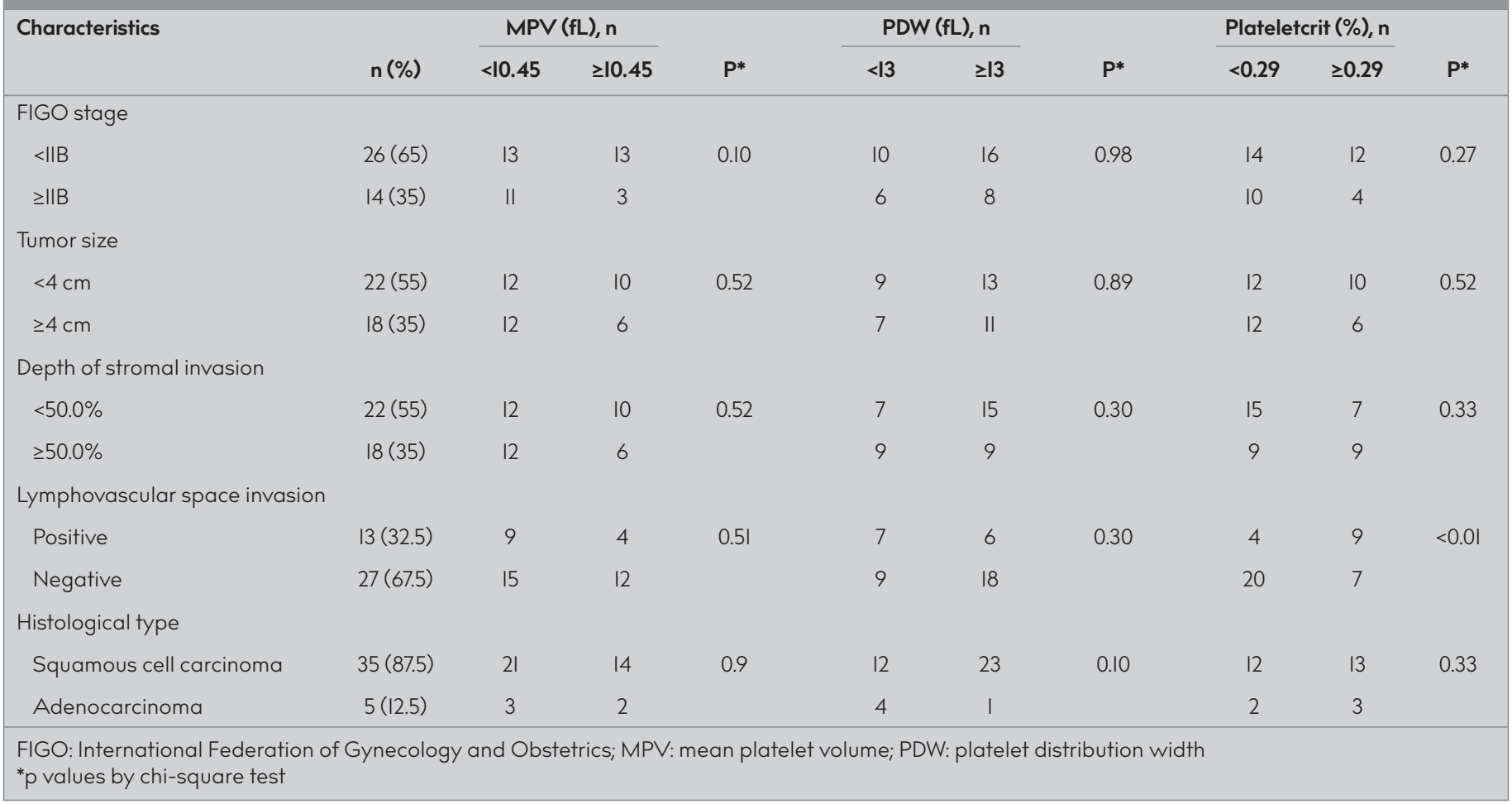


contrast to these studies, in the present study, we did not find a relationship between high platelet count and $\mathrm{CC}$. This may be a result of the relatively small sample size of our study, as well as a consequence of methodological differences between the studies (i.e., inclusion and exclusion criteria).

Mean platelet volume represents the average volume of platelets found in blood. PDW is an indicator of volume variability in platelet size, and its increase shows that abnormally large and small platelets are in the circulation. Both of them are an index of platelet activation and are associated with different inflammatory conditions. Studies with non-gynecological (i.e., gastric, colon, and lung cancers) and gynecological cancers (i.e., endometrial, ovarian, and CCs) revealed that both MPV and PDW may have differed significantly (i.e., increase or decrease) in patients with cancer compared with healthy controls $(3,20-22,24,25)$. Similar to these studies, in the present study, we did find that both MPV and PDW were significantly lower in patients with CC than in control patients. Nevertheless, studies investigating the relationships between these markers and pathological features of CC have yielded inconsistent results $(3,25)$. Similar to two previous studies, in the present study, we did not find any significant relationships between CC's pathological features (i.e., tumor stage and size, depth of stromal invasion, LVSI status, and histological type) and neither MPV nor PDW, respectively $(3,25)$.

Plateletcrit is the volume occupied by platelets in the blood as a percentage and is considered as analogous to hematocrit, which used to evaluate red cell mass. In healthy subjects, the range of PCT varies between $0.22 \%$ and $0.24 \%$, but has no clinical meaning by itself (26). However, limited studies with inflammatory diseases, coronary heart diseases, and various pregnancy-related conditions, such as preeclampsia and hyperemesis gravidarum, displayed that PCT may significantly correlate with the prognosis of these diseases (27-30). Furthermore, studies with various cancer types, such as lung and endometrial cancers, revealed that PCT increased significantly in these cancer types and was related with poor prognosis $(23,24)$. This may be the result of platelet number and functional changes that occurred as part of paraneoplastic syndrome. In the present study, PCT was significantly higher in patients with CC than in healthy controls, and high PCT level $(\geq 29.0 \%)$ was significantly correlated with positive LVSI status. To our knowledge, this is the first report that identified a relationship between PCT and CC.

In conclusion, although the present study has its limitations (e.g., its retrospective single-center design and relatively small sample size), it revealed that serum MPV, PDW, and PCT levels of $<10.35 \mathrm{fL},<13 \mathrm{fL}$, and $\geq 0.29 \%$, respectively, were significantly associated with CC. On the other hand, the present study revealed that except a significant relationship between high PCT level and positive LVSI, there was no significant relationship between Pls and CC's pathological features. However, further descriptive and comparative studies are needed to determine the clinical utility of Pls in the preoperative diagnosis of CC and its pathological features.

Ethics Committee Approval: Ethics committee approval was received for this study from the Ethical Review Board of Ankara Atatürk Training and Research Hospital. (Approval Date: II/7/2018, Approval Number: I17).
Informed Consent: Informed consent was obtained from the patients who participated in this study.

Peer-review: Externally peer-reviewed.

Author contributions: Concept - E.E.T.; Design - E.E.T.; Supervision - H.L.K., A.F.Y.; Resource - E.E.T., G.F.Y., E.A.K.; Materials - G.F.Y., E.A.K.; Data Collection and/or Processing - E.E.T., E.A.K.; Analysis and/or Interpretation - E.E.T., E.A.K., H.L.K., G.F.Y; Literature Search - G.F.Y., E.A.K.; Writing - E.E.T., G.F.Y., H.L.K., E.A.K.; Critical Reviews - A.F.Y.

Conflict of Interest: The authors have no conflicts of interest to declare.

Financial Disclosure: The authors declared that this study has received no financial support.

\section{REFERENCES}

I. Priebe AM. 2012 cervical cancer screening guidelines and the future role of HPV testing. Clin Obstet Gynecol 2013; 56: 44-50. [CrossRef]

2. Cheng J, Zeng Z, Ye Q, Zhang Y, Yan R, Liang $C$, et al. The association of pretreatment thrombocytosis with prognosis and clinicopathological significance in cervical cancer: a systemic review and meta-analysis. Oncotarget 2017; 8: 24327-36. [CrossRef]

3. Fu S, Niu Y, Zhang X, Zhang JR, Liu ZP, Wang RT. Squamous cell carcinoma antigen, platelet distribution width, and prealbumin collectively as a marker of squamous cell cervical carcinoma. Cancer Biomark 2018; 21: 317-21. [CrossRef]

4. Mantovani A, Allavena P, Sica A, Balkwill F. Cancer related inflammation. Nature 2008; 454: 436-44. [CrossRef]

5. Chen L, Zhang F, Sheng XG, Zhang SQ, Chen YT, Liu BW. Peripheral platelet/lymphocyte ratio predicts lymph node metastasis and acts as a superior prognostic factor for cervical cancer when combined with neutrophil: lymphocyte. Medicine (Baltimore) 2016; 95: e4381. [CrossRef]

6. Wang L, Jia J, Lin L, Guo J, Ye X, Zheng X, et al. Predictive value of hematological markers of systemic inflammation for managing cervical cancer. Oncotarget 2017; 8: 44824-32. [CrossRef]

7. Li X, Tan C, Zhang W, Zhou J, Wang Z, Wang S, et al. Correlation between platelet and hemoglobin levels and pathological characteristics and prognosis of early-stage squamous cervical carcinoma. Med Sci Monit 2015; 21: 392I-8. [CrossRef]

8. Kose M, CelikF, Kose SK, Arioz DT, Yilmazer M. Could the platelet-to-lymphocyte ratio be a novel marker for predicting invasiveness of cervical pathologies? Asian Pac J Cancer Prev 2015; 16: 923-6. [CrossRef]

9. Zhu M, Feng M, He F, Han B, Ma K, Zeng X, et al. Pretreatment neutrophil-lymphocyte and platelet-lymphocyte ratio predict clinical outcome and prognosis for cervical cancer. Clin Chim Acta 2018; 483: 296-302. [CrossRef]

10. Hoskin PJ, Rojas AM, Peiris SN, Mullassery V, Chong IY. Pre-treatment haemoglobin and peripheral blood lymphocyte count as independent predictors of outcome in carcinoma of cervix. Clin Oncol (R Coll Radiol) 2014; 26: 179-84. [CrossRef]

II. Lee YY, Choi CH, Sung CO, Do IG, Hub SJ, Kim HJ, et al. Clinical significance of changes in peripheral lymphocyte count after surgery in early cervical cancer. Gynecol Oncol 2012; 127: 107-I3. [CrossRef]

12. Mabuchi S, Matsumoto Y, Isohashi F, Yoshioka Y, Ohashi H, Morii E, et al. Pretreatment leukocytosis is an indicator of poor prognosis in patients with cervical cancer. Gynecol Oncol 20II; 122: 25-32. [CrossRef]

13. Martínez-Donate AP, Vera-Cala LM, Zhang X, Vedro R, Angulo R, Atkinson T. Prevalance and correlates of breast and cervical cancer screening among a Midwest community sample of low-acculturated Latinas. J Health Care Poor Underserved 2013; 24: 1717-38. [CrossRef]

14. Zhang Y, Wang L, Liu Y, Wang S, Shang P, Gao Y, et al. Preoperative neutrophil-lymphocyte ratio before platelet-lymphocyte ratio predicts clinical outcome in patients with cervical cancer treated with initial radical surgery. Int J Gynecol Cancer 2014; 24: 1319-25. [CrossRef] 
15. Zheng RR, Huang XX, Jin C, Zhuang XX, Ye LC, Zheng FY, et al. Preoperative platelet count improves the prognostic prediction of the FIGO staging system for operable cervical cancer patients. Clin Chim Acta 2017; 473: 198-203. [CrossRef]

16. Li S, Hu T, Lv W, Zhou H, Li X, Yang R, et al. Changes in prevalence and clinical characteristics of cervical cancer in the People's Republic of China: a study of 10.012 cases from a nationwide working group. Oncologist 2013; 18: II0I-7. [CrossRef]

17. National Cancer Institute: Surveillance Epidemiology and End Results: Cancer Stat Facts: cervix uteri. Available from: URL: https:// seer.cancer.gov/statfacts/html/cervix.html. (Accessed 16.09.2018)

18. Lorin L, Bertaut A, Hudry D, Beltjens F, Roignot P, Bone-Lepinoy MC, et al. About invasive cervical cancer: a French population based study between 1998 and 2010. Eur J Obstet Gynecol Reprod Biol 2015; 191: 1-6. [CrossRef]

19. Golebiewska EM, Poole AW. Platelet secretion: From haemostasis to wound healing and beyond. Blood Rev 2015; 29: 153-62. [CrossRef]

20. Klınıçalp S, Ekiz F, Bașar O, Ayte MR, Coban S, Yılmaz B, et al. Mean platelet volume could be possible biomarker in early diagnosis and monitoring of gastric cancer. Platelets 2014; 25: 592-4. [CrossRef]

21. Li JY, Li Y, Jiang Z, Wang RT, Wang XS. Elevated mean platelet volume is associated with presence of colon cancer. Asian Pac J Cancer Prev 20I4; 15: 1050I-4. [CrossRef]

22. Kemal Y, Demirağ G, Ekiz K, Yücel I. Mean platelet volume could be a useful biomarker for monitoring epithelial cancer. J Obstet Gynaecol 2014; 34: 515-8. [CrossRef]
23. Oncel M, Kiyici A, Oncel M, Sunam GS, Sahin E, et al. Evaluation of platelet indices in lung cancer patients. Asian Pac J Cancer Prev 2015; 16: 7599-602. [CrossRef]

24. Karateke A, Kaplanoglu M, Baloglu A. Relations of platelet indices with endometrial hyperplasia and endometrial cancer. Asian Pac J Cancer Prev 2015; 16: 4905-8. [CrossRef]

25. Shen WJ, Fu S, Li N, Li LL, Cao ZG, Li C, et al. Decreased mean platelet volume is associated with cervical cancer development. Asian Pac J Cancer Prev 2017; 18: 1769-72.

26. Budak YU, Polat M, Huysal K. The use of platelet indices, plateletcrit, mean platelet volume and platelet distribution width in emergency non-traumatic abdominal surgery: a systemic review. Biochem Med (Zagreb) 2016; 26: 178-93. [CrossRef]

27. Tang J, Gao X, Zhi M, Zhou HM, Zhang M, Chen HW, et al. Plateletcrit: a sensitive biomarker for evaluating disease activity in Crohn's disease with low hs-CRP. J Dig Dis 2015; I6: II8-24. [CrossRef]

28. Gul M, Uyarel H, Akgul O, Akkaya E, Surgit O, Cakmak HA, et al. Long-term prognostic significance of admission plateletcrit values in patients with non-ST elevation myocardial infarction. Blood Coagul Fibrinolysis 2016; 27: 696-70I. [CrossRef]

29. Tayfur C, Burcu DC, Gulten O, Betul D, Tugberk G, Onur O, et al. Association between platelet to lymphocyte ratio, plateletcrit and the presence and severity of hyperemesis gravidarum. J Obstet Gynaecol Res 2017; 43: 498-504. [CrossRef]

30. Singh A, Varma R. Role of platelet distribution width (PDW) and plateletcrit in the assessment of nonthrombocytopenic preeclampsia and eclampsia. J Obstet Gynaecol India 2018; 68: 289-93. [CrossRef] 